\title{
Wilson-Mikity-Syndrom als Ursache einer respiratorischen Insuffizienz bei einem Frühgeborenen
}

\author{
A. Hilgendorff', I. Reiss ${ }^{1}$, J. Thul' ${ }^{2}$, G. Alzen ${ }^{3}$, L. Gortner ${ }^{1}$ \\ 1 Zentrum für Kinderheilkunde und Jugendmedizin, Abt. Allgemeine Pädiatrie und Neonatologie, Justus-Liebig-Universität, Gießen \\ 2 Zentrum für Kinderheilkunde und Jugendmedizin, Abt. Kinderkardiologie, Justus-Liebig-Universität, Gießen \\ 3 Zentrum für Radiologie, Abt. Kinderradiologie, Justus-Liebig-Universität, Gießen
}

\section{Zusammenfassung}

Das Wilson-Mikity Syndrom als eine Differentialdiagnose der chronischen Lungenerkrankungen der Neonatalperiode betrifft in erster Linie Frühgeborene und zeichnet sich durch eine fehlende Anamnese hinsichtlich typischer klinischer und radiologischer Veränderungen des Atemnotsyndroms (RDS) aus. Infektiöse Ursachen werden diskutiert.

Wilson-Mikity Syndrome as a cause of respiratory insufficiency of prematurity

The Wilson-Mikity syndrome is a differential diagnosis of chronic lung disease in the neonate and primarily related to immaturity. It is characterized by the absence of typical clinical and radiological findings of the respiratory distress syndrome (RDS). Infectious causes are being discussed.

\section{Fallbericht}

Anamnese: Wir berichten über ein Frühgeborenes von $32+1$ SSW, Geburtsgewicht $1500 \mathrm{~g}$ (P 25), Spontangeburt aus klarem Fruchtwasser bei vorzeitiger Wehentätigkeit. Eine Lungenreifeinduktion mit Betamethason erfolgte zweimalig pränatal. Apgar 8/8/9, $\mathrm{NspH} 7$,3. Postnatal kam es zu einer raschen Adaptation ohne klinische Zeichen einer Atemstörung.

Bei Verdacht auf ein Amnioninfektionssyndrom wurde eine antibiotische Therapie begonnen. Weder klinisch, laborchemisch noch mikrobiologisch zeigten sich im weiteren Verlauf Zeichen einer perinatal erworbenen Infektion.

Aufnahmebefund: Unauffälliger körperlicher internistischer Befund, initiale $\mathrm{BGA}$ mit $\mathrm{pCO}_{2} 44 \mathrm{~mm} \mathrm{Hg}, \mathrm{pH} 7,4, \mathrm{BE} 2 \mathrm{mmol} / \mathrm{l}$, transkutane Sauerstoffsättigung $>95 \%$ bei Raumluft.

Verlauf: Am zweiten Lebenstag (LT) kam es zu einem zunehmenden Sauerstoffbedarf, um eine arterielle Sauerstoffsättigung $>90 \%$ zu erreichen, kombiniert mit deutlicher Tachypnoe. Es kam zu einer zunehmenden $\mathrm{CO}_{2}$-Retention mit $\mathrm{pCO}_{2} 72 \mathrm{~mm} \mathrm{Hg}, \mathrm{pH}$ 7,28, BE $5 \mathrm{mmol} / \mathrm{l}$, im Röntgenbild des Thorax am 3. Lebenstag zeigte sich eine grobnetzige, teils zystisch imponierende Zeichnungsvermehrung über beiden Lungenfeldern (Abb.1). Die $\mathrm{O}_{2}-$ Supplementation über eine Nasenbrille erfolgte vom 2. bis zum 15. LT (max 50\%), danach stabile Sauerstoffsättigung bei Raumluft.

Labordiagnostik: Ausschluss bakterieller Infektionen, konnataler Infektionen, insbesondere Zytomegalie-, sowie Mycoplasmenund Chlamydieninfektionen.

Therapie: Das Frühgeborene erhielt eine hochkalorische und volumenrestriktive enterale Ernährung, eine diuretische Therapie mit Furosemid sowie Gabe von Theophyllin. Eine Behandlung mit Erythromycin wurde bei Verdacht auf eine interstitielle Lungenerkrankung am 2. LT begonnen und für 21 Tage fortgeführt.

Weitere Diagnostik: Eine Echokardiographie war ohne pathologischen Befund. Im Verlauf wurden weitere Röntgenaufnahmen des
Thorax, zuletzt am 49. LT (Abb. 2) angefertigt, ergänzt durch ein HR-CT am 54. LT (Abb. 3).

Aktueller Verlauf: Das Kind konnte mit einem Gewicht von $2400 \mathrm{~g}$ am 58. LT mit altersentsprechendem neurologischen Befund, Eupnoe, einer Sauerstoffsättigung von >95\% (transkutan) bei Raumluft und normwertiger BGA entlassen werden.

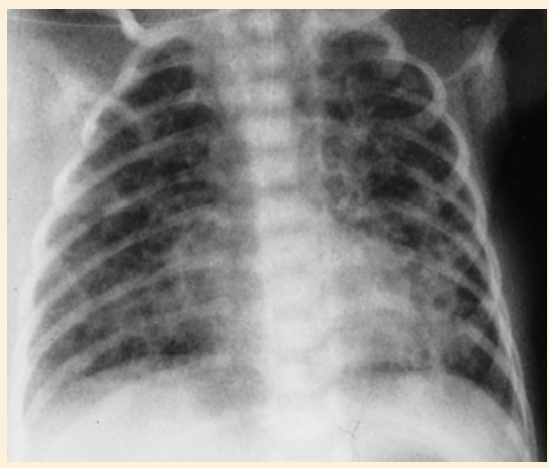

Abb. 1 RöntgenThorax am 3. Lebenstag in a.p. Projektion: Grobnetzige, teils zystisch imponierende Zeichnungsvermehrung über beiden Lungenfeldern.

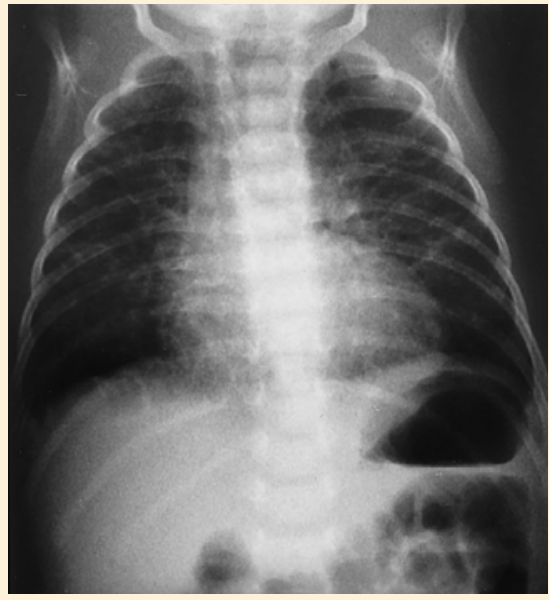

Abb. 2 RöntgenThorax am 49. Lebenstag in a.p.-Projektion: Interstitielle Herdzeichnung, im Verlauf diskret rückläufig. 


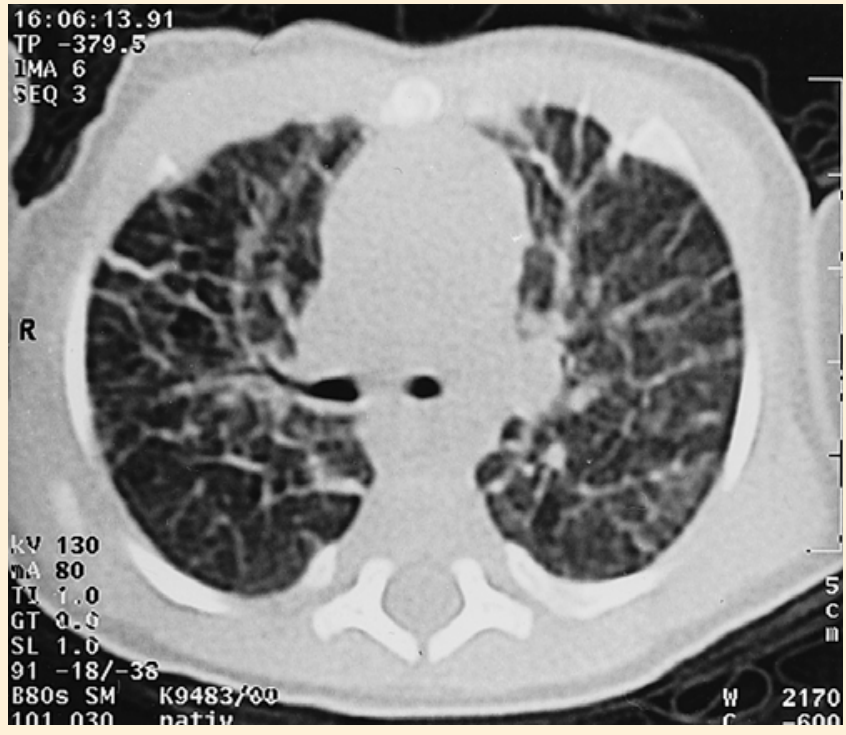

Abb. 3 CT-Schnitt im HR-mode bei Spontanatmung: massive Verdikkung der Interalveolarsepten. Deutlich vermehrte radiäre Struktur im Sinne einer ausgeprägten Fibrosierung.

\section{Diskussion}

Differenzialdiagnostik der chronischen Lungenerkrankung des Neugeborenen(CLD)

Die Differenzialdiagnosen der CLD sind in Tab. 1 aufgeführt.

In der Analyse des Röntgenbildes vom 3. LT stehen die Diagnosen der Bronchopulmonalen Dysplasie (BPD) und in Abgrenzung dazu das Wilson-Mikity-Syndrom im Vordergrund.

Bronchopulmonale Dysplasie: Die BPD ist gekennzeichnet durch einen persistierenden $\mathrm{O}_{2}$-Bedarf und Atemnotsymptomatik $>28$ Lebenstage und schließt charakteristische radiologische Veränderungen mit ein [1].

Ätiologisch sind eine maschinelle Beatmung bei Lungenunreife, insbesondere das Ergotrauma, die Sauerstofftoxizität, ein persistierender Ductus arteriosus Botalli und oder eine Flüssigkeitsüberladung sowie pulmonale oder systemische Infektionen zu eruieren [2]. Auch eine genetische Prädisposition erscheint möglich [3].

Im Hinblick auf Definition und Ätiologie der BPD lässt sich bei dem vorgestellten Frühgeborenen die Diagnose des WilsonMikity-Syndroms abgrenzen.

Wilson-Mikity-Syndrom: Erstbeschreibung des Krankheitsbildes im Jahre 1960 [4]. Betroffen sind meist Frühgeborene mit einem Gestationsalter von weniger als 32 Wochen und einem Geburtsgewicht unter $1500 \mathrm{~g}$, die die chronisch respiratorische Erkrankung in der Regel im postnatalen Alter von 10 bis 14 Tagen entwickeln.

Das wegweisende Kriterium ist der Röntgen-Thorax mit grobretikulären Infiltraten und Bezirken mit zystischen Veränderungen besonders in den Lungenoberfeldern [5].

Die pulmonale Symptomatik, die sich langsam progredient zeigt und mit Tachy-/Dyspnoe und oftmals erhöhtem $\mathrm{O}_{2}$-Bedarf einhergeht, persistiert in der Regel über Wochen bis Monate und ist klinisch sowie radiologisch schwer zu differenzieren von der BPD.
Tab.1 Differentialdiagnostik der chronischen Lungenerkrankung (CLD)

- bronchopulmonale Dysplasie

- Mekoniumaspiration

- diffuse neonatale Pneumonie

- zystische Fibrose

- pulmonales interstitielles Emphysem

- kongenitale pulmonale Lymphangiektasien

- idiopathische pulmonale Fibrose

- angeborene Herzfehlbildungen mit interstitieller Zeichnungsvermehrung

- Wilson-Mikity-Syndrom

Tab. 2 Differentialdiagnostisch wegweisende Befunde

- unauffälliger initialer pulmonaler Untersuchungsbefund

- Ausschluss eines Amnioninfektionssyndroms

- kein Chlamydien- und Mycoplasmennachweis

- Bakteriologie negativ

- negative Serologie der konnatalen Infektionen, insb. kein CMV-Nachweis

- echokardiographischer Ausschluss eines angeborenen Herzfehlers

Ätiologie: Eine endgültige Klärung der Pathogenese konnte bisher nicht erreicht werden. Ein ursächlicher Zusammenhang mit der pulmonalen Unreife, ungleichen Compliancewerten in den verschiedenen Lungenabschnitten oder einem infektiösen Agens wird diskutiert [6].

Therapie und Prognose: Die Therapie erfolgt symptomatisch analog zu den Therapienansätzen der BPD. Die Prognose ist im Allgemeinen sehr gut mit einer Rückbildung der Symptome (klinisch und radiologisch) innerhalb von ein bis zwei Jahren.

\section{Literatur}

${ }^{1}$ Northway WH, Rosan RC, Porter DY. Pulmonary disease following respirator therapy of Hyaline-membrane disease. Bronchopulmonary dysplasia. N Engl J Med 1967 276(7): 357-368

${ }^{2}$ Resch B, Eber E, Zach M. Chronic interstitial lung diseases in childhood: Bronchopulmonary dysplasia and exogenous allergic alveolitis. Klin Pädiatr 1998 210(5): 331 - 339

${ }^{3}$ Gortner L, Borkhardt A, Stoll-Becker S, Göpel W, Reiss I, Weber B. Association of Polymorphisms of Surfactant Protein A Genes and the Risk of BPD in Preterms with RDS. Ped Res 2000 47: 399A

${ }^{4}$ Kirkpatrick $\vee$ B, Mueller DG. Respiratory disorders in the newborn: Wilson-Mikity syndrome. Chernick and Boat: Disorders of the respiratory tract in children; 1998: 353-354

${ }^{5}$ Reittner P, Fotter R, Tillich M, Popper H, Lindbichler F, Reiterer F. High resolution $\mathrm{CT}$ findings in Wilson-Mikity syndrome: a case report. Pediatr Radiol 1998 28(9): 691 - 693

${ }^{6}$ Reiterer F, Dornbusch HJ, Urlesberger B, Reittner P, Fotter R, Zach M, Popper H, Muller W. Cytomegalovirus associated neonatal pneumonia and Wilson-Mikity syndrome: A causal relationship? Eur Respir ] 1999 13(2): $460-462$

\section{Dr. Irwin Reiss}

Zentrum für Kinderheilkunde und Jugendmedizin Abteilung Allgemeine Pädiatrie und Neonatologie Feulgenstr. 12, 35392 Gießen

Tel. 0641/9943400

Fax 0641/9943419

E-mail: irwin.reiss@paediat.med.uni-giessen.de 